\title{
Flexible photoanode on titanium foil for back-illuminated dye sensitized solar cells
}

\begin{abstract}
This paper reports the fabrication and analysis of flexible photoanode on titanium (Ti) foil for back-illuminated dye sensitized solar cells (DSSCs). Performance comparison with the solid state FTO glass based DSSC using the back-illumination and frond-illuminated techniques were also carried. During the fabrication process, the surface of Ti foil, had been treated with $\mathrm{H} 2 \mathrm{O} 2$ and doctor blade method was applied for deposition of the photoanode on substrates. The measurement results show that the fabricated DSSC with flexible photoanode has power conversion efficiency of $1.00 \%$ under back illuminated solar radiation of 1.5 A.M while DSSCs with solid state photoanodes have power conversion efficiency of $0.53 \%$ (backilluminated) and $2.22 \%$ (front-illuminated), respectively. The DSSC with flexible photoanode has better power conversion efficiency than the DSSC with solid-state photoanode under back-illumination condition. However, it is comparatively low from front illumination DSSC due to platinized counter electrode partially reflects light, while iodine in the electrolyte absorbs photons affects the performance.
\end{abstract}

Keyword: DSSC; Flexible solar cell; Ti foil; Back-illumination; Photoanode 